\title{
Cablenet System Analysis for Deployable Antenna in Ground Test Condition
}

\author{
Guoqiang You \\ Xi'an FANYI University, Xi'an, 710105, China \\ simonyouyou@163.com
}

\begin{abstract}
Keywords: Deployable antenna; Cablenet system; Ground test; Cable pretension; Catenary element; Gravity
\end{abstract}

\begin{abstract}
While deployable antenna works in space, there are little or no gravity effects on antenna's state, and it is no need to analyze antenna's cablenet system with considerations of gravity effects. But before launching, deployable antenna is to be assembled and tested in ground conditions, so in this situation, it is necessary to add gravity effects to antenna's analysis model to make sure the calculations are still accurate. For this sake, the catenary element that can simulate cable problems in conditions with gravity effects was used in this paper to build the cablenet system's finite element method model for deployable antenna. Firstly, formula of catenary element is deduced based on cable's original length. Then by using this type of cable element, the finite element method model of antenna's cablenet system in ground conditions is set up. And at last, this finite element method model is analyzed in an example, and the results show the difference of cablenet system between space conditions and ground conditions.
\end{abstract}

\section{Introduction}

Cablenet is increasingly being used in spaceborne deployable antenna system for its special qualities such as lighter weight, smaller collapsed volume and more flexible material. Cablenet can be used to form long-span reflector for spaceborne deployable antenna with strict shape precision requirement, and this is very different from its other usage in common buildings [1]. There is little or no gravity in space, but before launching, deployable antenna has to be manufactured and tested on the ground. So gravity effects on cablenet of deployable antenna in ground test are a problem we need to study.

Cablenet is a kind of flexible structure with geometric nonlinearity. Its cable elements need particular pretensions to obtain cablenet's given shape and stiffness. Cablenet problems can always be solved by methods such as nonlinear finite element method, balance matrix method, force density method and dynamic relaxation method. A lot of papers have studied the subject about analysis and calculation for cablenet of deployable antenna. In these papers, paper [2] summarized the difference of above methods when they are used for solving deployable antenna cablenet problems. Paper [3] used balance matrix method to analyze and optimize some different types of deployable antenna's cablenet, and gave out the optimal cable pretension distributions. Paper [4] used minimum norm method to optimize pretensions of a non-axisymmetric deployable antenna's cablenet, and obtained an optimal cable pretension distribution that can meet the demand for stress uniformity. Paper [5] used nonlinear finite element method to optimize pretensions of deployable antenna's cablenet with minimum reflector's shape precision as goal, and obtained the optimal structural parameters.

All above papers studied cablenet of deployable antenna without consideration of gravity effects. When antenna works in space, there are little or no gravity effects, but before launching, the antenna should be adjusted and tested in gravity environment, and in this situation, special attention need to be given to how much difference there will be for the cablenet's shape between these two kinds of environments. For this reason, Catenary element that can simulate cable in gravity environment is used to establish the finite element model of deployable antenna's cablenet system in conditions with gravity effects, and by solving this finite element model, we can obtain accurate shape and pretension 
distribution in ground environment, and make sure the difference between gravity effects and no gravity effects $[6,7]$.

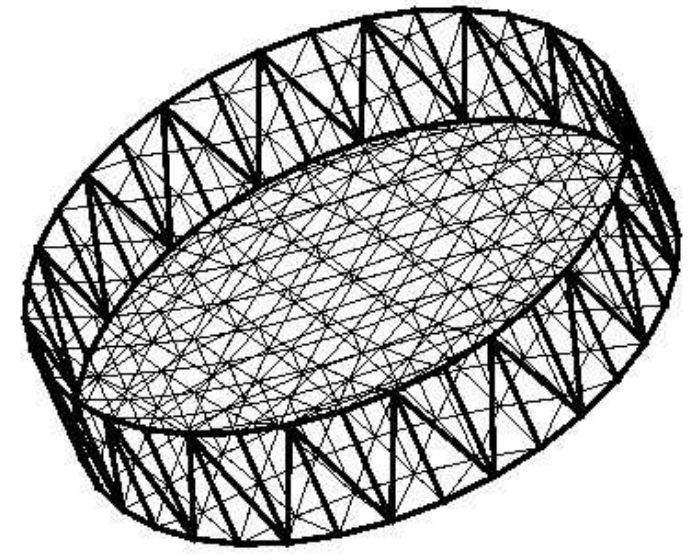

Figure 1. Finite Deployable antenna's structure

\section{Analysis for Deployable Antenna's Cablenet with Consideration of Gravity Effects}

For deployable antenna's cablenet with consideration of no gravity effects, seeing cable elements as two nodes line segments and using balance matrix method to linearly analyze it, the pretension value of every cable element in cablenet system can be accurately calculate [8,9]. But before launching, deployable antenna need to be tested and adjusted on the ground, and because of gravity, cable elements will sag more or less, and force directions at element's end nodes will not be on the same straight line. In this case, deployable antenna's cablenet may deviate from its given shape in conditions with gravity effects. In order to find out the accurate effects of gravity on cablenet's shape and inner forces, we use catenary elements to simulate upper and lower cables in the cablenet system, and two nodes cable-bar elements for vertical cables in the cablenet system.

When it is assumed that catenary element shown in Fig.2 can meet the demand for large deformation and small strain, and gravity evenly loads on the cable element $[10,11]$, we can calculate cable length from formulae below.

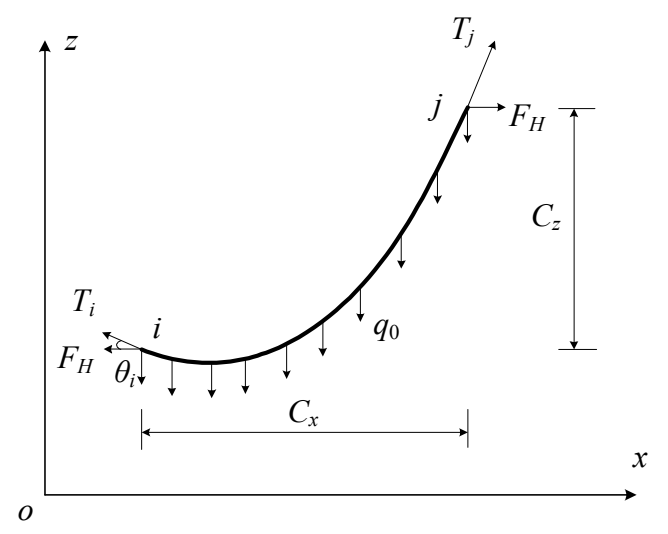

Figure 2. Finite Catenary element

$$
\begin{aligned}
& L^{2}=C_{x}{ }^{2} \frac{\sinh ^{2} \eta}{\eta^{2}}+C_{z}^{2} \\
& \eta=\frac{q_{0} C_{x}}{2 F_{H}}
\end{aligned}
$$

In above expressions, $L$ is cable length after loading, $C_{x}$ and $C_{z}$ are horizontal and vertical projected length of catenary chord, $q_{0}$ is cable weight per unit length, $F_{H}$ is horizontal tension on end node. 
The relationship between original cable length $L_{0}$ and horizontal tension $F_{H}$ can be written as,

$$
\begin{aligned}
& f\left(F_{H}\right)=\frac{4 F_{H}^{2}}{q_{0}^{2}} \sinh ^{2} \mu+\frac{C_{z}^{2}}{\left[1+\left(\frac{q_{0} L_{0}}{2 A E}\right) \operatorname{coth} \mu\right]^{2}}-L_{0}^{2}=0 \\
& \mu=\frac{q_{0}}{2}\left(\frac{C_{x}}{F_{H}}-\frac{L_{0}}{A E}\right)
\end{aligned}
$$

Getting the derivation of $F_{H}$ in Equ.(3), we can obtain equation below further.

$$
\frac{d f}{d F_{H}}=\frac{8 F_{H}}{q_{0}^{2}} \sinh ^{2} \mu-\frac{2 C_{x}}{q_{0}} \sinh 2 \mu-\frac{C_{x} L_{0}}{2 A E\left[1+\left(\frac{q_{0} L_{0}}{2 A E}\right) \operatorname{coth} \mu\right]^{3}}\left(\frac{C_{z} q_{0}}{F_{H} \sinh \mu}\right)^{2}
$$

Because cable's original length $L_{0}$ is known, we can use Newton-Raphson method to solve Euq.(5) to obtain cable node's $F_{H}$.

From $F_{H}$, we can get catenary element's nodal component force as shown below.

$$
\begin{aligned}
& F_{i x}=-F_{H} \\
& F_{i z}=F_{H} \tan \theta_{i}=\frac{q_{0}}{2}\left(L-C_{z} \frac{\cosh \eta}{\sinh \eta}\right) \\
& F_{j x}=F_{H} \\
& F_{j z}=q_{0} L_{0}-F_{i z}=q_{0} L_{0}-\frac{q_{0}}{2}\left(L-C_{z} \frac{\cosh \eta}{\sinh \eta}\right) \\
& T_{i}=\sqrt{{F_{i x}}^{2}+F_{i z}^{2}} \\
& T_{j}=\sqrt{{F_{j x}{ }^{2}+F_{j z}^{2}}^{2}}
\end{aligned}
$$

In above equations, $F_{i x}$ and $F_{i z}$ are horizontal and vertical component force of the $i$ th cable node in local coordinate system, $F_{j x}$ and $F_{j z}$ are horizontal and vertical component force of the $j$ th cable node, $T_{i}$ and $T_{j}$ are nodal tension of the $i$ th and the $j$ th cable node, $\theta_{i}$ is the angle between the cable element and horizontal direction at $i$ th cable node.

By using balance matrix method described in previous section, we can get the optimal cablenet pretension distribution with no consideration of gravity effects, and then we can use Equ.(12) and Equ.(13) below to calculate cables' original lengths in conditions with elasticity and small strain.

$$
\begin{aligned}
& L_{0}=L-\Delta L \\
& \Delta L=\frac{T}{A E} L
\end{aligned}
$$

$L_{0}$ is cable original length, $L$ is cable length with deformation caused by pretension, $\Delta L$ is elongation of cable element caused by pretension, $T$ is cable element's pretension calculated by balance matrix method.

When cable's original length and nodes' locations are known, we can obtain tangent stiffness matrix of catenary elements in conditions with gravity effects.

Furthermore, the vertical cables in deployable antenna's cablenet system have approximately the same direction with gravity, and this type of cable will stay straight line shape in conditions with gravity effects, so we use two nodes cable-bar element to simulate vertical cables for cablenet's FEM model.

Based on cablenet's original structural parameters in conditions with no gravity, and with catenary elements simulate upper and lower cables, two nodes cable-bar elements simulate vertical cables, we can establish non-linear Newton-Raphson iterative equations for antenna's cablenet system in conditions with gravity effects as shown below, 


$$
\Delta u^{i+1}=\left[\boldsymbol{K}_{\mathrm{T}}^{i}\right]^{-1} R^{i}
$$

In these equations, $\Delta u^{i+1}$ is displacement increment in the $i+1$ th step, $\boldsymbol{K}_{\mathrm{T}}{ }^{i}$ is the whole structure's tangent stiffness matrix in the $i$ th step, $R^{i}$ is node's residual force vector in the $i$ th step, $F^{i}$ and $P^{i}$ are $R^{i}=F^{i}-P^{i}$ node's external force vector and inner force vector.

$R^{i}=F^{i}-P^{i} \quad$ By solving above iterative equations, we can get nodal displacements and inner forces of deployable antenna's cablenet in conditions with gravity effects.

Furthermore, it is necessary to note that there is a layer of wire mesh put on antenna's cablenet system. And its weight should also be considered a kind of external force for the cablenet system.

\section{Numerical Experiment and Discussion}

Based on the method described in this paper, we used Fortran language to write the corresponding programs, and analyzed triangular mesh cablenet system of a deployable antenna with $10 \mathrm{~m}$ diameter and $6 \mathrm{~m}$ paraboloid focal length in conditions with gravity effects. The deployable antenna's cablenet system is shown in Fig .3. This cablenet system is made up of 439 cable elements. Based on axial symmetry of this triangular mesh cablenet system, we can divide the system into twelve groups with the same topology structure as shown in Fig.3, and it turns out that every group includes only 27 independent cable pretension variables. Symbols in Fig. 3 are pretension serial numbers of each cable element, and among these numbers, 20-27 are for vertical cables. And for this simplified cablenet system, a group of ideal cable pretentions shown in Table 1 can be obtained easily by using balance matrix method with the average value $\bar{T} \leq 15 \mathrm{~N}$ as constrain.
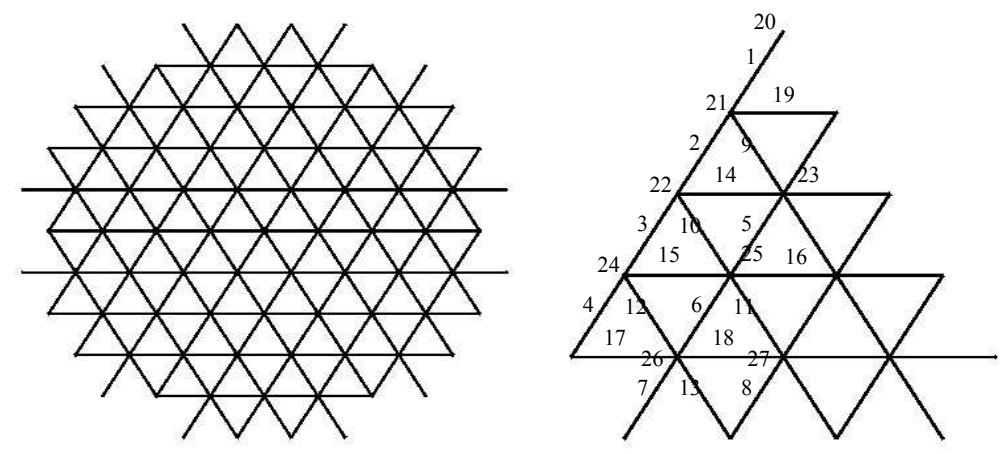

Figure 3. Finite Deployable antenna's cablenet system

Table 1 The given pretensions of cablenet

\begin{tabular}{|c|c|c|c|c|c|}
\hline Variable No. & Variable value & Variable No. & Variable value & Variable No. & Variable value \\
\hline$T_{1}$ & $20.4549[\mathrm{~N}]$ & $T_{10}$ & $19.0685[\mathrm{~N}]$ & $T_{19}$ & $17.7977[\mathrm{~N}]$ \\
\hline$T_{2}$ & $19.0576[\mathrm{~N}]$ & $T_{11}$ & $19.9018[\mathrm{~N}]$ & $T_{20}$ & $5.1093[\mathrm{~N}]$ \\
\hline$T_{3}$ & $19.3006[\mathrm{~N}]$ & $T_{12}$ & $19.4049[\mathrm{~N}]$ & $T_{21}$ & $4.7330[\mathrm{~N}]$ \\
\hline$T_{4}$ & $19.3534[\mathrm{~N}]$ & $T_{13}$ & $19.5276[\mathrm{~N}]$ & $T_{22}$ & $4.7275[\mathrm{~N}]$ \\
\hline$T_{5}$ & $19.5910[\mathrm{~N}]$ & $T_{14}$ & $18.9221[\mathrm{~N}]$ & $T_{23}$ & $4.7962[\mathrm{~N}]$ \\
\hline$T_{6}$ & $19.3397[\mathrm{~N}]$ & $T_{15}$ & $18.8906[\mathrm{~N}]$ & $T_{24}$ & $4.7253[\mathrm{~N}]$ \\
\hline$T_{7}$ & $19.9841[\mathrm{~N}]$ & $T_{16}$ & $18.2632[\mathrm{~N}]$ & $T_{25}$ & $4.7325[\mathrm{~N}]$ \\
\hline$T_{8}$ & $20.2953[\mathrm{~N}]$ & $T_{17}$ & $18.9969[\mathrm{~N}]$ & $T_{26}$ & $4.7376[\mathrm{~N}]$ \\
\hline$T_{9}$ & $19.3914[\mathrm{~N}]$ & $T_{18}$ & $19.0630[\mathrm{~N}]$ & $T_{27}$ & $4.8345[\mathrm{~N}]$ \\
\hline
\end{tabular}

By using catenary elements to simulate upper and lower cables, two nodes cable-bar elements for vertical cables, we analyze above cablenet system in conditions with gravity effects. In this case, cable's weight per unit length is set to $4.6181 \mathrm{E}-2 \mathrm{~N} / \mathrm{m}$, cable density is set to $1.5 \mathrm{E} 3 \mathrm{~kg} / \mathrm{m}^{3}$, elastic 
modulus of cable element is set to $1.24 \mathrm{E} 11 \mathrm{~N} / \mathrm{m} 2$ and cable radius is set to $1 \mathrm{~mm}$. It is necessary to note here that for gravities of vertical cables, they are added to the ends of cable-bar elements just as a kind of external load. After using non-linear finite element method to calculate this model, we obtain that root mean square error of all cablenet's unrestrained nodes' displacements is $2.5105 \mathrm{E}-2 \mathrm{~mm}$ in its self-balance state. When the average nodal force of catenary element is considered its inner force, the root mean square of differences between catenary cables' inner forces and pretension values in Table 1 is $0.6130 \mathrm{~N}$.

Above results suggest that gravity make lower cables' inner forces reduce a little and make upper cables' inner forces increase a little. But in general, for deployable antenna, gravity effects of cable elements are very weak for cables' small cross-sectional areas and light weights.

In view of the weight of wire mesh reflector put on antenna's upper cables, it is necessary to add its gravity as nodal external load to cablenet's FEM model. In this paper, the wire mesh's mass is set to $5 \mathrm{Kg}$, and after it is added to the non-linear finite element calculation, we obtain that root mean square error of all cablenet's unrestrained nodes' displacements is $6.9439 \mathrm{E}-2 \mathrm{~mm}$. The root mean square of differences between cables' inner forces and pretension values in Table 1 is $1.7020 \mathrm{~N}$. These results show that though wire mesh's weight is small, its effects on inner force change of cablenet need careful attention. And for antenna's cablenet system, a large inner force change will have adverse effects on its deployment property.

\section{Conclusions}

Firstly, by using catenary elements to simulate upper and lower cables, two nodes cable-bar elements to simulate vertical cables, the deployable antenna's cablenet FEM model in ground conditions is established and analyzed. The results show that for antenna's cablenet system, there is only very little shape difference between gravity state and no gravity state, so we can consider that shape precision of cablenet system in ground test is equal to it in space.

Secondly, at the end of the example, we set the weight of wire mesh as external load and add it to the calculation, and results show that the cablenet's shape does not have a measurable change by this external load. But in this case, the root mean square of cablenet's inner force change rises to $1.7020 \mathrm{~N}$, and for the antenna's cablenet system, it is necessary to bring attention to this problem for a potential risk of worse deployment property.

\section{Acknowledgements}

Financial supports by Shaanxi Province Department of Education(16JK2076) are gratefully acknowledged.

\section{References}

[1] M. Koryo and M. Yasuyuki, Concept of the tension truss antenna, AIAA Journal. 28 (1990) 1098-1104.

[2] S. Pellegrino and C. R. Calladine, Matrix analysis of statically and kinematically indeterminate framworks, International Journal of Solids \& Structures. 22 (1986) 409-428.

[3] G. Li and F. Guan, Pretension optimization in parabolic cable net of Astromesh deployable reflector, Journal of Zhejiang University. 39 (2005) 1557-1560. (In Chinese)

[4] D. Yang and H. Bao, Force balance characteristics and pretension design of asymmetric cable net parabolic antenna, Journal of Mechanical Engineering. 45 (2009) 308-312. (In Chinese)

[5] J. Di, B. Duan and Y. Luo, etc, Preloading optimization of large net-shape deployable antennas, Journal of South China University of Technology. 32 (2004) 23-26. (In Chinese)

[6] J. Mitsuji and T. Yasaka, Shape control of the tension truss antenna, AIAA. 28 (1990) 316-322.

[7] M. Misawa, Stiffness design of deployable satellite antennas in deployed configuration, Int. J. Spacecraft and Rockets. 35 (1998) 380-386. 
[8] J. Huang, The development of inflatable array antennas, IEEE Transaction on Antennas and Propagation. 43 (2001) 44-50.

[9] T. Takano et. al, Deployable antenna with 10-m maximum diameter for space use, IEEE Transactions on Antennas and Propagation. 52 (2004) 2-11.

[10]M. Kaminski and B. Schrefler, Probabilistic effective characteristics of cables for super conducting coils, Computer Methods in Applied Mechanics and Engineering. 188 (2000) 1-16.

[11]N. Vassart and R. Motro, Multiparametered formfinding method: Application to tensegrity systems, International Journal of Space Structures. 14 (1999) 147-154. 